\title{
SNP regulation of miRNA Expression and its association with Osteoporosis
}

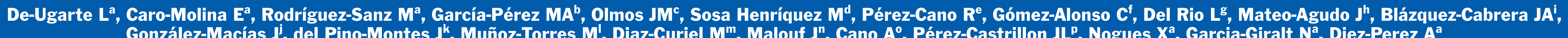
IIMIM (Hospital del Mar Medical Research Institute), Universitat Autonoma de Barcelona, Barcelona. RETICEF (ISCII); 'Department of Genetics and Institute of Health Research INCLIVA, University of Valencia, Valenciaa; 'Department of Internal

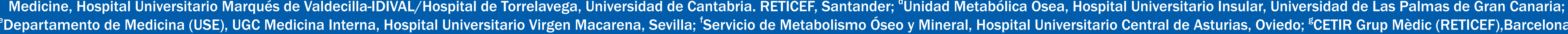
'Servicio COT Hospital Universitario Miguel Servet, Zaragozoza; 'Complejo Hospitalario Universitario de Albacete, Albacete; 'Departamento de Medicicina Interna, H. Marqués de Valdecilla, Universidadad de Cantabria, IDIVAL, RETICEF, Santander;

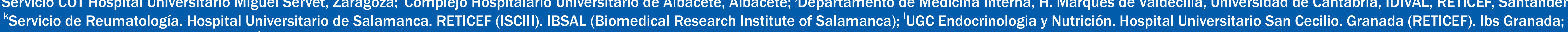
"Unidad de Enfermedades Metabólicas Óseas. Servicio de Medicina Interna. Fundacion Jimenez Diaz, Madrid;" "Hospital de la Santa Creu i Sant Pau. Institut d'Illnvestigació Biomèdica Sant Pau, Barcelona; 'Department of Pediatrics, Obstetrics and Gynecology and Institute of Health Research INCLIVA, University of Valencia; ${ }^{\mathrm{P}}$ Hospital Universitario Río Hortega, Valladolid

\section{Introducction}

miRNAs have been extensively studied in bone research, particularly their relationship to osteoporosis (De-Ugarte et al, BMC Med Genomics, 2015; Seeliger et al, J Bone Min Research, 2014) . However, the miRNA expression signatures described in patients with osteoporosis do not provide evidence of causality because the altered pattern could be a consequence of the disease or even unrelated to the pathogenesis. Another approach in miRNAs studies is the association analysis between
one SNP within a candidate miRNA (miR-SNP) or in a miRNA target site, and one disease related-outcome. In this case, the associated variant is likely involved in the pathophysiology or confers susceptibility to develop the disease (Estrada et al, Nat Genet, 2012).

\section{Objectives}

The aims of this study were (1) to identify SNPs within candidate density (BMD), the main outcome used to define osteoporosis and (2) to validate in bone cells this miR-SNP association with the osteoporotic phenotype.

\section{Methods}

SNPs located in pre-miRNA sequences that (1) bind to the MRNA 3'UTR of genes related with bone metabolism or (2) found to be highly expressed in human osteoblasts were selected. Validated SNPs with a MAF $>0.01(n=5)$ were genotyped in the OSTEOMED2 cohort (Table 1$)$ to assess their association. with LS BMD and FN BMD (Table 3). Multivariate linear regression models were fitted to assess the association between genotyped SNPs and BMD. Potential confounders considered for adjustment were densitometer devices, body mass index (BMI) and age.

PiRNAs which harbored BMD-associated SNPs were quantified by GPCR in order to compare the expression levels between OP and non-OP bone samples (Table 2). Mann-Whitney $U$ test was performed for $O P$ and non-OP group comparisons.

Human primary osteoblasts were cultured for DNA and RNA extraction and sorted by genotype for both rs6430498 and rs12512664. The correlation 列 All analyses were two-tailed, and p-values $<0.05$ were considered significant.

Table 1. Baseline characteristics of the OSTEOMED2 cohort.

\begin{tabular}{lcc} 
Patient characteristic & \multicolumn{2}{c}{ Mean \pm SD } \\
\cline { 2 - 3 } & LS BMD & FN BMD \\
& $n=2183$ & $n=2015$ \\
Age (years) & $57.61 \pm 9.26$ & $58.80 \pm 8.99$ \\
Age of menopause (years) & $48.7 \pm 3.94$ & $48.7 \pm 3.92$ \\
BMI $\left(\mathbf{k g} / \mathbf{m}^{2}\right)$ & $26.56 \pm 4.18$ & $26.48 \pm 4.13$ \\
BMD $\left(\mathrm{g} / \mathrm{cm}^{2}\right)$ & $0.870 \pm 0.16$ & $0.707 \pm 0.14$ \\
\hline Abbreviations: BMI=body mass index; BMD=bone mineral density; \\
LS=lumbar spine; FN=femoral neck
\end{tabular}

\begin{tabular}{|c|c|c|c|c|}
\hline & $n$ & $\begin{array}{c}\text { Age } \\
\text { (Mean } \pm \text { SD) }\end{array}$ & $\begin{array}{l}\mathrm{BMI}\left(\mathrm{kg} / \mathrm{m}^{2}\right) \\
(\text { Mean } \pm \mathrm{SD})\end{array}$ & $\begin{array}{l}\mathrm{BMD}\left(\mathrm{g} / \mathrm{cm}^{2}\right) \\
(\mathrm{Mean} \pm \mathrm{SD})\end{array}$ \\
\hline $\begin{array}{l}\text { Biological groups } \\
\text { Osteoporotic }\end{array}$ & 10 & $75.6 \pm 6.38$ & $27.11 \pm 2.94$ & Fragility fracture \\
\hline Non-osteoporotic & 10 & $71.7 \pm 7.36$ & $27.42 \pm 3.15$ & $0.882 \pm 0.137$ \\
\hline
\end{tabular}

\section{Results}

SNP rs6430498 in the miR-3679 and rs12512664 in the miR-4274 were significantly associated with FN BMD (Table 4). The A alleles for rs6430498 (5)

Table 4. SNPs associated with FN BMD in linear-regression analysis

\begin{tabular}{|c|c|c|c|c|c|}
\hline SNP ID & miRNA & $\begin{array}{l}\text { Genotyping } \\
\text { efficiency (\%) }\end{array}$ & HWE & $\begin{array}{l}\text { Beta coefficient }{ }^{\mathrm{a}} \\
\quad[95 \% \mathrm{Cl}]\end{array}$ & $p$ value \\
\hline $\begin{array}{l}\text { rs6430498 } \\
\text { rs12512664 }\end{array}$ & $\begin{array}{l}\text { miR-3679 } \\
\text { miR-4274 }\end{array}$ & $\begin{array}{l}97.96 \\
98.72\end{array}$ & $\begin{array}{c}1 \\
0.47\end{array}$ & $\begin{array}{c}-0.017[-0.032 \text { to }-0.003] \\
0.015[0.004 \text { to } 0.027]\end{array}$ & $\begin{array}{l}0.021^{R} \\
0.01^{R}\end{array}$ \\
\hline
\end{tabular}

Adjusted for DXA device, age and BMI

Both miRNAs miR-3679-3p and miR-4274 were significantly overexpressed in the OP samples (Table 5).

\begin{tabular}{|c|c|c|c|c|}
\hline \multicolumn{6}{|c|}{ Table 5. miRNA expression levels, comparison between osteoporotic and non-osteoporotic bone samples } \\
\hline miRNA & Biological Group & RQ (Median) & IQR & P value \\
\hline miR-3679 & Osteoporotic & 89.601 & 220.636 & 0.001 \\
& Control & 1.423 & 0.664 & \\
miR-4274 & Osteoporotic & 144.268 & 318.409 & 0.001 \\
& Control & 1.197 & 2.154 & \\
\hline
\end{tabular}

Abbreviations: RQ=Relative quantification: IQR=Interquartile Range

A significant correlation was observed between miRNA levels and the genetic variant (Figure 1). The A allele for both SNPs was associated with higher expression of each corresponding miRNA (miR-3674; log-additive model; $p$-value $=0.015$, and miR-4274; dominant model; $p$-value $=0.013$ ). Additionally, circumstances, another boneelated miRNAs were checked in these cells and no differences in expression were found irrespective of genotypes.

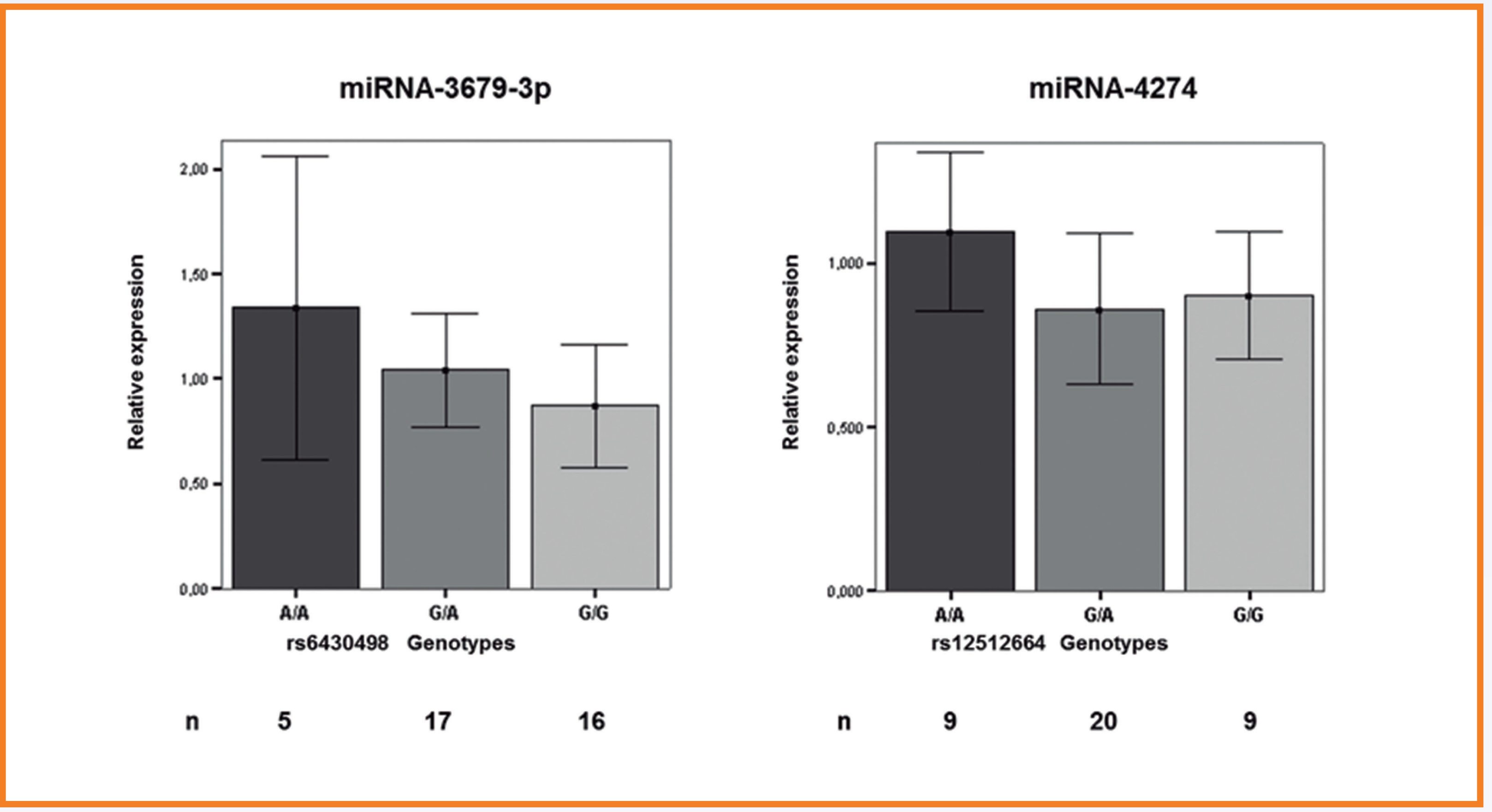

Figure 1. Correlation between miRNA expression levels and genotypes for miR-3679 (1) expression in Real-Time PCR. U6 was used for normalization. Samples of 38 human primary osteoblasts were used for experiments. (n) is the number of samples for each genotype group.
Table 3. Validation of miR-SNPs for the BMD association analysis.

\begin{tabular}{|c|c|c|c|}
\hline TARGET GENE & miRNA & SNP & MAFB \\
\hline \multirow[t]{10}{*}{ ESR1 } & miR-106b & rs72631827 & not polym. \\
\hline & miR-130b & rs72631822 & not polym. \\
\hline & miR-148b & rs74878365 & not polym. \\
\hline & miR-18a & rs41275866 & not polym. \\
\hline & miR-222 & rs72631825 & not polym. \\
\hline & miR-373 & rs80338016 & not polym. \\
\hline & miR-520c & rs7255628 & not polym. \\
\hline & miR-93 & rs72631824 & not polym. \\
\hline & miR-96 & rs41274239 & 0.0033 \\
\hline & & rs73159662 & 0.0058 \\
\hline \multirow{15}{*}{ TGFB2 } & miR-141 & rs34385807 & not polym. \\
\hline & miR-149 & rs71428439 & not polym. \\
\hline & miR-182 & rs77586312 & not polym. \\
\hline & & rs75953509 & not polym. \\
\hline & & rs80041074 & 0.0033 \\
\hline & miR-199b & rs72631835 & not polym. \\
\hline & miR-193a & rs60406007 & not polym. \\
\hline & miR-200b & rs72563729 & not polym. \\
\hline & miR-33a & rs77809319 & not polym. \\
\hline & miR-431 & rs76090066 & 0.00083 \\
\hline & & rs128840'05 & not polym. \\
\hline & miR-590 & rs6971711 & not polym. \\
\hline & miR-7-1 & rs76662330 & not polym. \\
\hline & miR-7-2 & rs41276930 & 0.005 \\
\hline & & rs75737367 & not polym. \\
\hline \multirow[t]{3}{*}{ PTH1R } & miR-339 & rs13232101 & not polym. \\
\hline & & rs72631820 & not polym. \\
\hline & & rs72631831 & not polym. \\
\hline \multirow[t]{2}{*}{ RUNX2 } & miR-122 & rs41292412 & 0.0033 \\
\hline & miR-154 & rs41286570 & 0.0004 \\
\hline \multirow[t]{7}{*}{ IL6R } & miR-124-2 & rs72631829 & not polym. \\
\hline & miR-124-3 & rs34059726 & not polym. \\
\hline & miR-125a & rs12975333 & not polym. \\
\hline & miR-140 & rs7205289 & not polym. \\
\hline & miR-320d-1 & rs74826059 & not polym. \\
\hline & miR-499 & rs3746444 & 0.21 \\
\hline & & rs7267163 & 0.0025 \\
\hline LRP5 & miR-27a & rs11671784 & 0.0162 \\
\hline \multirow[t]{4}{*}{ IL6 } & miR-146a & rs2910164 & 0.26 \\
\hline & miR-146b & rs76149940 & not polym. \\
\hline & miR-202 & rs12355840 & not polym. \\
\hline & miR-365-2 & rs35143473 & not polym. \\
\hline \multirow[t]{2}{*}{$V D R$} & miR-10a & rs72631828 & not polym. \\
\hline & miR-223 & rs34952329 & not polym. \\
\hline \multirow[t]{5}{*}{ CYP24A1 } & miR-30b & rs111424617 & not polym. \\
\hline & miR-30e & rs112439044 & not polym. \\
\hline & miR-183 & rs72631833 & not polym. \\
\hline & & rs41281222 & not polym. \\
\hline & miR-101-2 & rs78851134 & 0.0004 \\
\hline \multirow[t]{2}{*}{ Highly expressed } & miR-1282 & rs11269 & not polym. \\
\hline & miR-3679 & rs6430498 & 0.35 \\
\hline \multirow[t]{2}{*}{ in HObs } & & rs10175383 & not polym. \\
\hline & miR-4274 & rs12512664 & 0.47 \\
\hline
\end{tabular}

MAFB; Minor allele frequency in BARCOS cohort
In bold; Validated SNPS for genotyping in total OSTEOMED2 cohort

\section{Conclusions}

- Genetic variants in miR-3679 and miR-4274 are associated with femoral neck bone mineral density. In both cases, alleles associated with lower BMD correlated with higher expression levels of respective mature miRNAs in human osteoblastic cells which were found overexpressed in fractured bone samples.

- Our results open new exploratory avenues for future studies in the bone field and the treatment of osteoporosis.

\section{References}

1. De-Ugarte L, Yoskovitz G, Balcells S, Guerri-Fernandez R, Martinez-Diaz S, Mellibovsky L, Urreizti R, Nogues X, Grinberg D, Garcia-Giralt N et al: MiRNA profiling of whole trabecular bone: identification of osteoporosis-related changes in MiRNAs in human hip bones. BMC Med Genomics 2015, 8(1):75.

2. 2.Seeliger C, Karpinski K, Haug A, Vester H, Schmitt A, Bauer J, van Griensven M: Five Freely Circulating miRNAs and Bone Tissue miRNAs are Associated with Osteoporotic Fractures. J Bone Miner Res 2014. Nat Genet 2012, 44(5):491-501. 